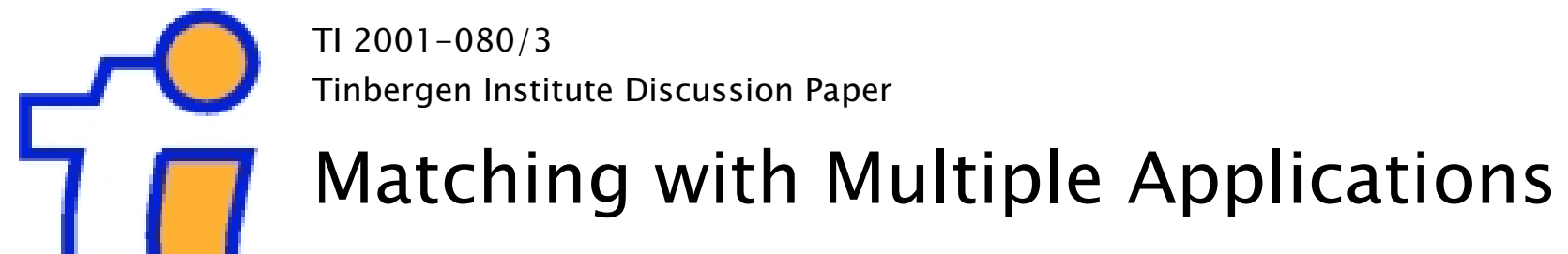

James W. Albrecht ${ }^{1}$

Pieter A. Gautier ${ }^{2}$

Susan B. Vroman'

' Georgetown University, Washington DC

2 Erasmus University Rotterdam, and Tinbergen Institute 
Tinbergen Institute

The Tinbergen Institute is the institute for economic research of the Erasmus Universiteit Rotterdam, Universiteit van Amsterdam and

Vrije Universiteit Amsterdam.

Tinbergen I nstitute Amsterdam

Keizersgracht 482

1017 EG Amsterdam

The Netherlands

Tel.: +31.(0)20.5513500

Fax: $\quad+31 .(0) 20.5513555$

Tinbergen Institute Rotterdam

Burg. Oudlaan 50

3062 PA Rotterdam

The Netherlands

Tel.: $\quad+31 .(0) 10.4088900$

Fax: $\quad+31 .(0) 10.4089031$

Most TI discussion papers can be downloaded at

http://www.tinbergen.nl 


\title{
Matching with Multiple Applications
}

\author{
James W. Albrecht* \\ Pieter A. Gautier ${ }^{\dagger}$ \\ Susan B. Vroman
}

August 27, 2001

\begin{abstract}
We analyze the implications of multiple applications by job seekers for the microfoundations of the aggregate matching function. We emphasize a coordination failure caused by multiple applications that has not been previously considered, namely, that firms can waste time and effort processing an applicant who is ultimately hired by another firm.
\end{abstract}

Keywords: search, matching

JEL codes: J41, J64, D84

*Department of Economics, Georgetown University, Washington DC; albrecht@georgetown.edu. $\dagger^{\dagger}$ Erasmus University Rotterdam and Tinbergen Institute Amsterdam; gautier@tinbergen.nl

*Department of Economics, Georgetown University, Washington DC; vromans@georgetown.edu. 


\section{Introduction}

In the process of hiring new assistant professors, a problem faced by many departments is that the candidate they make an offer to is hired by another department. This costs time and money in added recruitment effort. Departments rarely face a problem of having no acceptable applicants. Nonetheless, in the leading microfoundations model for the matching function, the urn-ball model, the matching friction that is modeled is the latter rather than the former. In this note, we extend the urn-ball model to allow for multiple applications by job seekers. This extension highlights the inefficiency that arises when firms waste resources processing applicants who are ultimately hired by other firms.

The aggregate matching function is central to the theoretical literature on unemployment (Pissarides 2000). Several papers, starting with Butters (1977) and Pissarides (1979), have used the urn-ball model from probability theory to provide microfoundations for this aggregate function. The basic assumption in the urn-ball model is that each worker (ball) applies randomly to one vacancy (urn). There is a coordination failure because each worker does not know where other workers are applying. As a result, some vacancies receive no applicants and remain unfilled while others receive multiple applications. The inefficiency is that some vacancies that could be productively filled are not, while at the same time some workers who could be productively employed are not.

In this note, we incorporate an essential feature of the matching process that is not present in the basic urn-ball model, namely, that workers apply to more than one job at a time. One reason that workers do this is because it takes time for firms to process applications. Incorporating multiple applications per worker into the matching process results in a new coordination problem because more than one firm can process the same worker, but only one is able to hire the worker. Thus, some vacancies remain unfilled because their applicants are hired elsewhere. As departments who have lost their preferred candidates to other institutions know, this coordination failure is important in the real world. $^{1}$

\footnotetext{
${ }^{1}$ In another context, it is interesting to note that in economics, a norm has evolved that forbids
} 
There are alternatives to urn-ball models as microfoundations for the matching function. These include the stock/flow matching model of Coles (1994) and Coles and Smith (1998) and the directed search model of Lagos (2000). Neither of these models captures the coordination externality that arises from multiple applications in our model. In addition to theoretical models of the matching function, there is also an extensive empirical literature. Petrongolo and Pissarides (2001) give an overview of both literatures. They conclude that a majority of estimates for different countries and different periods show that there is a stable constant returns to scale matching function. Further, this function is often of the Cobb Douglas form. We show that in a special case, our matching function can serve as a microfoundation for a Cobb Douglas matching function.

In the next section, we introduce multiple applications by searching workers and processing time by firms into a basic urn-ball model and derive the matching function. We relate our result to the usual urn-ball matching function and discuss the properties of our function. Section 3 discusses extensions of our matching process, including the special case that gives rise to a Cobb Douglas matching function. Section 4 contains conclusions.

\section{Matching with multiple applications}

We consider a labor market in steady-state. At any time, there are $u$ unemployed workers and $v$ vacancies, and each unemployed worker has $a$ applications outstanding. The application process is undirected in the sense that any application is equally likely to go to any one of the vacancies, but the individual does not apply more than once to any particular vacancy. Firms process applications one at a time, and it takes one "period" to evaluate an application. The urn-ball model is the special case of $a=1$.

The matching function can be expressed in two equivalent ways, $M(u, v)=v \times$ the probability per period that a vacancy is filled or $M(u, v)=u \times$ the probability per period that an unemployed worker finds a job. We attack the problem from the vacancy point of

consideration of the same article by more than one journal at the same time, thus solving this coordination problem. At law reviews, however, where articles are refereed by students rather than by faculty, multiple submissions are allowed. 
view. Thus, we need to derive the probability per period that a vacancy is filled, which we denote $\psi$.

Let $N$ be the number of applications that a firm has in hand. $N$ can take on the values $n=0,1, . ., u$. The probability that any one unemployed worker has applied to a particular vacancy is $\frac{a}{v}$, so $N$ is $\operatorname{bin}\left(u, \frac{a}{v}\right)$. The probability that a firm has an application to consider is $p \equiv 1-\left(1-\frac{a}{v}\right)^{u}$. When $a=1$, a firm with an applicant necessarily succeeds in hiring that applicant; that is, if $a=1$,

$$
M(u, v)=v p=v\left[1-\left(1-\frac{1}{v}\right)^{u}\right] .
$$

This is the standard urn-ball matching function.

If $a>1$, we need to account for the fact that a firm processing an applicant may lose that applicant to one of its rivals. To do this, consider a firm that is processing an application, i.e., $n>0$. The number of other firms that are simultaneously processing the same applicant is a random variable $Y$ that can take on the values $y=0,1, . ., a-1$. Let $q$ be the probability that the applicant is being considered by any one of the other $a-1$ vacancies to which he has applied, so $Y$ is $\operatorname{bin}(a-1, q)$.

Note that $q=\frac{v p}{a u}$. The derivation is as follows. The number of other applicants for any one of the other $a-1$ vacancies is a random variable $Z$, which is $\operatorname{bin}\left(u-1, \frac{a}{v}\right)$. Conditional on $Z=0$, the applicant is being considered for the other vacancy with probability 1 , conditional on $Z=1$, she is being considered with probability $\frac{1}{2}, \ldots$, conditional on $Z=u-1$, she is being considered with probability $\frac{1}{u}$. That is,

$$
q=\sum_{z=0}^{u-1} \frac{1}{z+1}\left(\begin{array}{c}
u-1 \\
z
\end{array}\right)\left(\frac{a}{v}\right)^{z}\left(1-\frac{a}{v}\right)^{u-1-z} .
$$

Let $s=z+1$. Then

$$
\begin{aligned}
q & =\sum_{s=1}^{u} \frac{1}{s}\left(\begin{array}{c}
u-1 \\
s-1
\end{array}\right)\left(\frac{a}{v}\right)^{s-1}\left(1-\frac{a}{v}\right)^{u-s}=\frac{v}{a u} \sum_{s=1}^{u}\left(\begin{array}{l}
u \\
s
\end{array}\right)\left(\frac{a}{v}\right)^{s}\left(1-\frac{a}{v}\right)^{u-s} \\
& =\frac{v}{a u}\left(\sum_{s=0}^{u}\left(\begin{array}{c}
u \\
s
\end{array}\right)\left(\frac{a}{v}\right)^{s}\left(1-\frac{a}{v}\right)^{u-s}-\left(\frac{a}{v}\right)^{0}\left(1-\frac{a}{v}\right)^{u}\right) \\
& =\frac{v}{a u}\left[1-\left(1-\frac{a}{v}\right)^{u}\right]=\frac{v p}{a u} .
\end{aligned}
$$


If $Y=0$, the firm "wins the race" for this applicant with probability 1, if $Y=1$, the firm wins with probability $\frac{1}{2}, \ldots$., if $Y=a-1$, the firm wins with probability $\frac{1}{a}$. The probability that a firm evaluating an applicant succeeds in hiring that applicant is thus

$$
\gamma=\sum_{y=0}^{a-1}\left(\frac{1}{y+1}\right)\left(\begin{array}{c}
a-1 \\
y
\end{array}\right) q^{y}(1-q)^{a-1-y}=\frac{1}{a q}\left[1-(1-q)^{a}\right] .
$$

The derivation is analogous to the one above, and we thus have

$$
\psi=p \gamma=\frac{p}{a q}\left[1-(1-q)^{a}\right] .
$$

Our aggregate matching function is then

$$
M(u, v)=v \psi=u\left[1-(1-q)^{a}\right]=u\left[1-\left(1-\frac{v}{a u}\left(1-\left(1-\frac{a}{v}\right)^{u}\right)\right)^{a}\right] .
$$

It is instructive to consider some special cases.

1. When $a=1, M(u, v)=v\left[1-\left(1-\frac{1}{v}\right)^{u}\right]$, i.e., the urn-ball matching function.

2. When $a=v, M(u, v)=u\left[1-(1-q)^{v}\right]=u\left[1-\left(1-\frac{1}{u}\right)^{v}\right]$.

3. Letting $u, v \rightarrow \infty$ with $\frac{v}{u}=\theta, M(u, v)=u\left[1-\left(1-\frac{\theta}{a}\left(1-\exp \left(-\frac{a}{\theta}\right)\right)\right)^{a}\right]$.

When $a=1$ and we let $u, v \rightarrow \infty$ with $\frac{v}{u}=\theta, M(u, v)=u \theta\left[1-\exp \left\{-\frac{1}{\theta}\right\}\right]$, and when $a=v$ and we let $u, v \rightarrow \infty$ with $\frac{v}{u}=\theta, M(u, v)=u[1-\exp \{-\theta\}]$. The symmetry between the $a=1$ and $a=v$ cases is interesting to note, as is the fact that in the limit as $u, v \rightarrow \infty$ with $\frac{v}{u}=\theta, M(u, v)$ exhibits CRS, irrespective of $a$. That is, in the limiting case, $M(u, v ; a)=u m(\theta ; a)$. We can also show that $\partial m(\theta ; a) / \partial \theta>0$, as is assumed in most theoretical matching models. We have

$$
\frac{\partial m(\theta ; a)}{\partial \theta}=\left[1-\frac{\theta}{a}\left(1-\exp \left(-\frac{a}{\theta}\right)\right)\right]^{a-1}\left[1-\exp \left(-\frac{a}{\theta}\right)-\frac{a}{\theta} \exp \left(-\frac{a}{\theta}\right)\right],
$$

which is positive so long as $1-\exp \left(-\frac{a}{\theta}\right)>\frac{a}{\theta} \exp \left(-\frac{a}{\theta}\right)$. To see this, note that $1-e^{-x}-x e^{-x}=$ 0 when $x=0$ and that the derivative of this function with respect to $x$ is positive for $x>0$. 
Several authors, e.g. Autor (2001), have suggested that the Internet should increase the efficiency of the matching process. The argument is that the Internet makes it easier to apply to many vacancies in a short period of time. More applications per worker reduces the urn-ball coordination problem, but there is a countervailing effect from multiple applications. Specifically, the more other jobs that a firm's applicant is simultaneously pursuing, the smaller is the probability that the firm in question will hire that applicant. The comparative statics of the matching function with respect to $a$ are thus of interest. We have investigated these comparative statics numerically for the limiting case of $u, v \rightarrow \infty$ with $\frac{v}{u}=\theta$. For $\theta$ ranging from 0.1 to 2 , we find that $\psi=p \gamma$ first increases but then decreases with $a$. For $a \geq 10$, the matching probability is close to constant. The idea that the Internet makes matching easier is thus not obvious.

\section{Extensions}

We could extend this analysis in several directions. One possibility is to assume that applicant productivity is a match-specific random variable $X$ with distribution function $F(x)$, independently and identically distributed across worker-job pairs. Suppose the firm rejects an applicant if $x \leq x^{*}$, and let $\xi=1-F\left(x^{*}\right)$. We take $x^{*}$ (and thus $\xi$ ) to be an exogenous constant, but this cutoff productivity could be endogenized, e.g., it could be made a function of $a$ and $\theta$.

As in the case of $\xi=1, N$ is $\operatorname{bin}\left(u, \frac{a}{v}\right)$ and $p=1-\left(1-\frac{a}{v}\right)^{u}$. Now, however, we need to take into account not how many rivals a firm has for an applicant but rather how many rivals the firm has that find that applicant qualified. Call this random variable $M$, and note that $M$ is $\operatorname{bin}(a-1, \xi q)$. Using a derivation analogous to that in the previous section, the probability that no other firm finds the applicant qualified and at the same time "wins the race" for that applicant is $\frac{1}{a \xi q}\left[1-(1-\xi q)^{a}\right]$. Multiplying this probability by $\xi p$, i.e., by the probability that the firm has an applicant to consider and that it finds the applicant acceptable, gives the probability that a particular vacancy is filled in a period 
of time. Then,

$$
M(u, v)=u\left[1-\left(1-\frac{\xi v}{a u}\left(1-\left(1-\frac{a}{v}\right)^{u}\right)\right)^{a}\right] .
$$

In the limit, as $u, v \rightarrow \infty$ with $\frac{v}{u}=\theta$, we have

$$
M(u, v)=u\left[1-\left(1-\frac{\xi \theta}{a}\left(1-\exp \left\{-\frac{a}{\theta}\right\}\right)\right)^{a}\right]
$$

As expected, the number of matches increases if firms are less selective, i.e., $\partial M(u, v ; \xi) / \partial \xi>$ 0.

Another interesting extension, which has a more dramatic effect on the matching function, is to consider the firm's processing time to be a continuous random variable. Suppose, for example, that the time required to complete the processing of an application is an exponential random variable with parameter $\phi .^{2}$ Consider a firm that is processing an application. As before, the number of other firms that are simultaneously processing the same applicant is a random variable $Y$ that can take on the values $y=0,1, . ., a-1$. To derive the aggregate matching function, we need to derive the expected time that it takes to fill the vacancy.

First note that as before if $y=0$, the firm "wins the race" for this applicant with probability 1 , if $y=1$, the firm wins with probability $\frac{1}{2}, \ldots$, if $y=a-1$, the firm wins with probability $\frac{1}{a}$. If $y=0$, the processing time is exponential with parameter $\phi$. If $y=1$, the time it takes to fill the vacancy (at the firm that wins the race) is exponential with parameter $2 \phi$ (because the minimum of two $\exp \{\phi\}$ random variables is $\exp \{2 \phi\}$ ), but the firm in question wins the race with probability $\frac{1}{2}$, etc.

A vacancy can be in any one of the following "states":

1. No applicant under consideration - this state occurs with probability $1-p$

2. An applicant under consideration with $y=0$ (i.e., no competition from other firms) - this state occurs with probability $p P[Y=0]$. A vacancy in this state results in a filled job at rate $\phi$ with probability 1 .

\footnotetext{
${ }^{2}$ We assume once again that $\xi=1$.
} 
3. An applicant under consideration with $y=1$ (i.e., the applicant is being considered by one other firm) - this state occurs with probability $p P[Y=1]$. A vacancy in this state results in a filled job at rate $2 \phi$ with probability $\frac{1}{2}$.

$\mathrm{a}+1$. An applicant under consideration with $y=a-1$ (i.e., the applicant is being considered by $a-1$ other firms) - this state occurs with probability $p P[Y=a-1]$. A vacancy in this state results in a filled job at rate $a \phi$ with probability $\frac{1}{a}$.

Since we want to derive the matching function in a steady-state world, we can think about the distribution of vacancies across states "cross-sectionally"; i.e., at any instant in time, a fraction $1-p$ of all vacancies is in state 1 , a fraction $p P[Y=0]$ is in state 2, etc. Alternatively, we could think about the distribution across states through time for a typical job, when vacant. In steady-state, these two distributions are the same. This means that we can compute the rate at which a typical vacancy is filled by taking a weighted sum of the rates at which vacancies in different states are filled with weights equal to the probabilities of being in the state in question. Since in all states, other than state 1 , the expected rate at which a job is filled is $\phi$ and since the probabilities associated with states $2, \ldots, n+1$ sum to $p$, we conclude that the rate at which a typical vacancy is filled equals $\phi p$.

That is, we conclude that the matching function is

$$
M(u, v)=v \phi p=v \phi\left[1-\left(1-\frac{a}{v}\right)^{u}\right]
$$

and, if we let $u, v \rightarrow \infty$ with $v / u=\theta$,

$$
M(u, v)=v \phi\left(1-\exp \left(-\frac{a}{\theta}\right)\right)
$$

As in the case with fixed processing time, this matching function exhibits CRS.

An important implication of the assumption of exponential processing time is that the coordination inefficiency associated with multiple applications disappears. The probability that a firm will lose the applicant it is processing to one of its rivals is increasing in 
the number of other firms that are considering that applicant, but when the firm replaces its applicant with a new candidate, the expected time until the vacancy is filled does not change. This memoryless property is, of course, unique to the exponential, and if one assumes another processing time distribution, the multiple applications coordination inefficiency is not eliminated.

To explore some of the other implications of the exponential, it is again instructive to consider special cases.

1. When $a=1, M(u, v)=v \phi\left[1-\exp \left(-\frac{1}{\theta}\right)\right]$, the same function as before, i.e., the urn-ball matching function, with the scale factor $\phi$.

2. When $a=v, M(u, v)=\phi v$. In this case, the first coordination problem is also absent because all vacancies receive applications. The matching function simply reduces to the processing rate times the stock of vacancies. Note that there is no congestion in this case. ${ }^{3}$ It is also interesting to note that this matching function exhibits CRS, even when $u$ and $v$ are small.

Perhaps a more interesting feature of the exponential assumption is that it can be used to generate a Cobb Douglas matching function. This is a useful exercise because the Cobb Douglas form is so commonly used despite the lack of any microfoundations. To derive the Cobb Douglas in this framework, let $\phi=\phi(\theta)$ and $a=v$. Substituting this into the matching function in the exponential case gives

$$
M(u, v)=v \phi(\theta)\left[1-\left(1-\frac{a}{v}\right)^{u}\right]=v \phi(\theta) .
$$

To generate the Cobb Douglas function, we need $v \phi(\theta)=A \theta^{\alpha}$. This holds if $\phi(\theta)=A \theta^{-\alpha}$, so that

$$
M(u, v)=v \phi(\theta)=v A \theta^{-\alpha}=A v^{1-\alpha} u^{a} .
$$

\footnotetext{
${ }^{3}$ In Gautier (2001), the labor market is modelled as a queueing system with directed search in the sense that workers always join the shortest queue (urn-ball problems are assumed away). In that model, contrary to here, more applications per worker only result in more congestion and unemployment.
} 
Why might $\phi(\theta)$ be a decreasing convex function of $\theta$ ? Van Ours and Ridder (1982) give evidence that what we call processing time is shorter the greater is the flow of applicants. That is, for given $v$, the processing rate, $\phi$, increases in $u$. Theoretically, however, it is not obvious why this should be the case. One possibility might be an IRS processing technology. Alternatively, one might fix $u$ and argue directly that $v \phi(\theta)$ is increasing and concave in $v$. For example,this could result from limited processing capacity in the economy, i.e., as $v$ increases, more workers get screened but at a decreasing rate. Of course, the assumptions on $\phi(\theta)$ that are required to reach this result are debatable, as is the assumption that processing time is exponential.

\section{Conclusions}

The contribution of this note has been to work out the implications of multiple applications by unemployed job seekers. Simultaneous applications are obviously common in real labor markets, and it turns out that allowing for this phenomenon has an important effect on the aggregate matching function. Specifically, allowing for multiple applications introduces a previously unconsidered coordination failure into the matching process - firms waste time and effort processing applicants who ultimately take a job with another firm. This inefficiency is distinct from and in addition to the standard urn-ball coordination failure. As the number of applications per unemployed worker, $a$, increases, the urn-ball coordination failure diminishes in importance, but the inefficiency due to multiple applications becomes more severe. In the limit, these two tendencies cancel, so the aggregate matching function is unaffected by further increases in $a$. This suggests that once $a$ is large enough, the Internet, which presumably makes multiple applications easier, should have little effect on the aggregate matching function.

In addition to exhibiting a new coordination failure, we show that the aggregate matching function is well-behaved when we allow for multiple applications. Specifically, as $u, v \rightarrow \infty$ with $v / u=\theta$, we show that it exhibits constant returns and that the matching rate for unemployed workers is increasing in $\theta$. In this sense, our aggregate matching func- 
tion is a natural generalization of the one derived from urn-ball microfoundations. We also consider some extensions, one of which allows for exponential processing time. In this case, the inefficiency caused by multiple applications is eliminated. While the exponential assumption is not, in our opinion, particularly plausible, this extension has the virtue of suggesting a possible microfoundations for Cobb Douglas matching.

\section{$5 \quad$ References}

Autor D. (2001), Wiring the labor market, Journal of Economic Perspectives, 15, 35-40.

Butters G. (1977), Equilibrium distributions of sales and advertising prices, Review of Economic Studies, 44, 465-491.

Coles M. (1994), Understanding the matching function: The role of newspapers and job agencies, CEPR discussion paper, No. 938.

Coles M. AND E. SMith (1998), Marketplaces and matching, International Economic Review, 39, 239-254.

Gautier P.A. (2001), Non-sequential search, screening externalities and the public good role of recruitment offices, forthcoming in Economic Modeling.

LAgos R. (2000), An alternative approach to search frictions, Journal of Political Economy, 108, 851-873.

Petrongolo B. And C.A. Pissarides (2001) Looking into the blackbox: A survey of the matching function, forthcoming Journal of Economic Literature.

Pissarides, C.A. (1979), Job matching with state employment agencies and random search, Economic Journal, 89, 818-33.

Pissarides, C.A. (2000) Equilibrium Unemployment Theory, Second Edition, MIT Press, Cambridge, MA.

VAn Ours, J.C. And G. Ridder (1982), Vacancies and the recruitment of new employees, Journal of Labor Economics, 10, 138-155. 